\title{
DOENTE SEDADO, CONSCIENTE E VENTILADO INVASIVAMENTE: TERAPÊUTICAS DE ENFERMAGEM
}

\author{
Sedated, conscious and invasively ventilated patient: nursing therapeutics \\ Enfermos sedados, conscientes y ventilados invasivamente: terapéutica de enfermería
}

\author{
Celeste Mata*, Maria Fernandes**, Maria Monteiro***, Odete Morais****, Susana Castro*****, Fernanda \\ Príncipe ${ }^{* * * * * *}$, Liliana Mota*******
}

\section{RESUMO}

Enquadramento: a sedação dos doentes sob ventilação invasiva tem sofrido uma mudança de paradigma, traduzida na utilização de protocolos de sedação ligeira, que possibilite que os mesmos estejam conscientes, sempre que possível. Os enfermeiros, através da implementação de terapêuticas de enfermagem adequadas têm papel relevante nesta transição saúde-doença. Objetivo: identificar as terapêuticas de enfermagem valorizadas pelos enfermeiros de Cuidados Intensivos, no cuidado ao doente ventilado invasivamente, sedado e consciente. Metodologia: estudo qualitativo, exploratório, mediante um focus group, com recurso à análise de conteúdo segundo Bardin. Amostra constituída por seis enfermeiros peritos, especialistas em enfermagem médico-cirúrgica, a exercer, em Serviços de Cuidados Intensivos de quatro hospitais do norte de Portugal. Resultados: emergiram cinco categorias de terapêuticas de enfermagem: redimensionamento da vigilância, gestão da terapêutica, autocuidado (com cinco subcategorias), implementação de estratégias comunicacionais e implementação de estratégias face à agitação/desorientação (com três subcategorias). Conclusões: as terapêuticas de enfermagem à pessoa sedada, consciente e ventilada invasivamente, valorizadas pelos enfermeiros de cuidados intensivos, focam-se no incremento da vigilância dos doentes, na gestão da analgesia/sedação, na promoção do potencial de autocuidado, na implementação de estratégias facilitadoras da comunicação e de estratégias para gestão dos quadros de agitação/desorientação.

Palavras chave: cuidados de enfermagem; cuidados críticos; sedação consciente; respiração artificial.

*RN, Enfermeira do Centro Hospitalar e Universitário do Porto - https://orcid.org/00000003-0605-7035 - Contribuição no artigo: Study conception and design, Data analysis and interpretation, Critical revision of the article

**RN Enfermeira do Centro Hospitalar Unive Universitário do Porto htps.//orcid.org/00000001-9978-2679 - Contribuição no artigo: Study conception and design, Data collection, Data analysis and interpretation, Drafting of the article ${ }^{* * *} \mathrm{RN}$, Enfermeira do Centro Hospitalar e Universitário do Porto - https://orcid.org/00000003-3769-1012 - Contribuição no artigo: Study conception and design, Data collection, Data conalysis and interpretation, Drafting of the article analysis and interpretation, Drafting of the article Universtaio do Port Universitário do Porto - https://orcid.org/0000 0003-0163-848X - Contribuição no artigo: Study conception and design, Data collection, Data analysis and interpretation, Drafting of the article *****RN, Enfermeira do Centro Hospitalar Universitário do Porto - https://orcid.org/00000003-0388-4115 - Contribuição no artigo: Study conception and desinn Data collection, Sata conception and desien, Data colection, Date analysis and interpretation, Drafting of the article * Escola Superior de Saúde Norte da Cruz Vermelha Portuguesa/CINTESIS. - https://orcid.org/0000 0002-1142-3258 Contribuição no artigo: Study conception and design, Data analysis and interpretation, Critical revision of the article

******* PhD Professor Adjunta da Escol Superior de Sáde Norte da Cruz Vermela Puperior de Saude Portuguesa/CINTESIS. - https://orcid.org/00000003-3357-7984 - Contribuição no artigo: Study conception and design, Data collection, Data analysis and interpretation, Drafting of the article, Critical revision of the article

\section{Como referenciar:}

Mata, C., Fernandes, M.F.., Monteiro, M.F.., Morais, O., Castro, S., Príncipe, F., \& Mota, L., (2021). Doente sedado, consciente e ventilado invasivamente: terapêuticas de enfermagem. Revista de Investigação \& Inovação em Saúde, 4(1) 7-17 https://doi.org/10.37914/riis.v4i1.118

Recebido para publicação em: 01/03/2021 Aceite para publicação:26/05/2021

\section{ABSTRACT}

Background: the sedation of patients under invasive ventilation has undergone a paradigm shift, translated into the use of light sedation protocols, which allows them to be aware, whenever possible. Nurses, through the implementation of appropriate nursing therapeutics, play an important role in the occurrence of this health-disease transition. Objectives: to identify nursing therapeutics valued by Intensive Care nurses in caring for patients with invasive ventilation, sedated and conscious. Methodology: qualitative, exploratory study, starting a focus group meeting, using content analysis according to Bardin. The sample consisted of six expert nurses, specialists in medical surgical nursing, working in Intensive Care Services at four hospitals in northern Portugal. Results: emerge five categories of nursing therapeutics: redimensioning of surveillance, management of therapy, self-care(with five subcategories), implementation of communication strategies and implementation of strategies in the face of agitation/disorientation( with three subcategories). Conclusions: nursing therapeutics for sedated, conscious and invasively ventilated people, valued by intensive care nurses, focus on increasing patient surveillance, in the management of analgesia/sedation, in promoting the potential for self-care, implementing strategies to facilitate communication and strategies for managing agitation/disorientation.

Keywords: nursing care; critical care; conscious sedation; respiration, artificial.

\section{RESUMEN}

Marco contextual: la sedatión de pacientes bajo ventilatión invasiva há sufrido un cambio de paradigma, traducido en el uso de protocolos de sedatión ligera, que les permite estar atentos, siempre que sea posible. Esta situatión es desafiante par las enfermeras, quienes, através de la implementatión de terapias de enfermería adecuadas, son relevantes en una transisión saludable salud-enfermedad. Objetivo: identificar las terapias de enfermería valoradas por los enfermeros de Cuidados Intensivos en el cuidado de pacientes ventilados invasivamente, sedado y consciente. Metodologia: estudio cualitativo, exploratorio, a partir de una reunión de grupo focal, utilizando análisis de contenido según Bardin. La muestra estuvo formada por seis enfermeras expertas, especialistas en enfermería médico-quirúrgica, desempeñando funciones en los Servicios de Cuidados Intensivos de cuatro hospitales del norte de Portugal. Resultados: surgen cinco categorías de terapias de enfermería: redimensionamiento de la vigilancia, manejo de la terapia, autocuidado (con cinco subcategorías), implementación de estrategias de comunicación e implementación de estrategias ante la agitación/desorientación (con tres subcategorías). Conclusiones: las terapias de enfermería para personas sedadas, conscientes y ventiladas de forma invasiva valorado por enfermeras de cuidados intensivos, se centran en aumentar la vigilancia del paciente, en el manejo de la analgesía/sedación, en la promoción del potencial del autocuidado, en la implementación de estrategias facilitadores de la comunicación y estrategias para manejar la agitación/desorientación.

Palabras clave: cuidados de enfermería; cuidado crítico; sedación consciente; respiración artificial 


\section{INTRODUÇÃO}

O internamento num Serviço de Cuidados Intensivos constitui uma transição marcante no estado de saúdedoença, de um indivíduo. O mesmo encontra-se numa situação de saúde crítica, ingressando, subitamente, num ambiente despersonalizado, altamente tecnológico, sendo submetido a uma panóplia de técnicas invasivas. Um dos procedimentos frequentes é a ventilação invasiva, associada ao controle da dor e sedação. Ao longo dos anos, a sedação dos doentes sob ventilação invasiva evoluiu no sentido da criação de protocolos de sedação ligeira, possibilitando que os mesmos estejam conscientes, sempre que a sua condição o permita. Cuidar de doentes neste contexto constitui um desafio para os enfermeiros (Tingsvik, Bexell, Andersson, \& Henricson, 2013), requerendo competências teóricas, técnicas, comunicacionais e psicossociais. A implementação de terapêuticas de enfermagem adequadas, focadas numa abordagem integral, individualizada e humanizada, são cruciais para que a transição do doente se realize satisfatoriamente. O objetivo deste estudo foi: identificar as terapêuticas de enfermagem valorizadas pelos enfermeiros de Cuidados Intensivos, no cuidado ao doente com ventilação invasiva, sedado e consciente.

\section{ENQUADRAMENTO/FUNDAMENTAÇÃO TEÓRICA}

A vida de cada indivíduo é pautada por mudanças e acontecimentos ímpares que, em muitos casos se revelam verdadeiramente desafiantes. Na perspetiva de Meleis (2010), a forma como cada indivíduo reage e se adapta a essas mudanças ou transições, não depende somente da sua individualidade, mas também da influência da família e do meio no qual se encontra inserido, no momento. Uma transição é o passar de um estado, condição ou lugar estável, para outro igualmente estável, o que implica, por parte da pessoa, a integração de conhecimentos, alteração de comportamentos e mudança da perspetiva do seu "eu". Este processo é singular, complexo, multidimensional, e, de acordo com a sua natureza, pode ser do tipo organizacional desenvolvimental, situacional e saúde/doença (Meleis, Sawyer, Im, Hilfinger, Messias, \& Schumacher, 2000).

A ocorrência de uma transição relacionada com o estado de saúde/doença é caracterizada como um evento crítico na vida de um indivíduo. Nesta linha de pensamento, o internamento num Serviço de Cuidados Intensivos constitui uma transição marcante para o indivíduo que, de uma forma abrupta se vê privado da sua saúde e da sua individualidade (Meleis et al., 2000). Quando ocorre a necessidade de internamento num Serviço de Cuidados Intensivos, verifica-se que os doentes aí admitidos, apresentam uma situação de doença crítica, em que a “(...) vida está ameaçada por falência ou eminência de falência de uma ou mais funções vitais e cuja sobrevivência depende de meios avançados de vigilância, monitorização e terapêutica" (Regulamento no 429/2018 da Ordem dos Enfermeiros). Estes, de uma forma imprevisível vêm-se inseridos num ambiente estranho, fragilizados e dependentes de pessoas que não conhecem, expostos e com pouca privacidade. As suas necessidades mais básicas são asseguradas, maioritariamente por dispositivos, que os substituem em funções tão básicas como a respiração, alimentação, eliminação e autocuidados. Concomitantemente, são alvo de múltiplas intervenções invasivas, que afetam quer a sua vertente física, quer a psíquica. Neste momento de maior 
Doente sedado, consciente e ventilado invasivamente: terapêuticas de enfermagem

vulnerabilidade e sofrimento, é crucial o papel desempenhado pelos enfermeiros de cuidados intensivos na transição do doente, da situação de doença crítica para a sua recuperação. Compete a estes profissionais aplicar as suas competências, conhecimentos e experiência, de modo a colmatar as dificuldades e necessidades do binómio doente/família, ajudando-os na aquisição de novas competências. É essencial que os enfermeiros de cuidados intensivos procurem envolver o doente e família nos cuidados, por forma a que ocorra consciencialização, adaptação e aceitação perante a doença, a fim de conseguirem ajustar-se a novos papeis, através do desenvolvimento de estratégias de coping (Meleis et al., 2000), alinhadas com a nova realidade. Desta forma, através da implementação de terapêuticas de enfermagem, tornase possível para os enfermeiros, planear os cuidados, assim como selecionar as intervenções adequadas, conducentes a uma transição saudável (Meleis 2010). Segundo esta autora (citada por Mota, Bastos, \& Brito, 2018, p. 21) "as terapêuticas de enfermagem incluem as intervenções e os objetivos das intervenções" e “(...) podem ser situadas no âmbito preventivo, promocional ou interventivo". Num ambiente em que a componente tecnológica é dominante, os conhecimentos e capacidades técnicas dos enfermeiros são enfatizados como essenciais para a estabilidade fisiológica do doente. É, no entanto, fulcral que estes profissionais de cuidados intensivos, para além das competências teóricas e técnicas, abarquem no seu processo de cuidar, as competências comunicacionais e psicossociais, proporcionando cuidados integrais, com dedicação, respeito e humanização. Compreender as determinantes do processo de transição possibilita o desenvolvimento de intervenções de enfermagem dirigidas para experiência única de cada pessoa e família, promovendo respostas saudáveis (Meleis et al., 2000).

Em Portugal, os enunciados descritivos dos cuidados de enfermagem especializados em pessoa em situação crítica, englobam a satisfação do cliente, promoção da saúde, prevenção de complicações, bem-estar e autocuidado, readaptação funcional, organização dos cuidados e prevenção e controlo da infeção associada aos cuidados (Ordem dos Enfermeiros, 2017). A responsabilidade dos enfermeiros de Cuidados Intensivos, pautada por uma natureza dinâmica, passa por intervenções conducentes à promoção, prevenção, proteção, alívio e minimização/resolução dos problemas decorrentes do estado de saúde crítico do doente, não descurando a família, enquanto interveniente em todo este processo.

No decurso da sua prática profissional, os enfermeiros que exercem funções em serviços de Cuidados Intensivos, deparam-se frequentemente com doentes submetidos a ventilação mecânica invasiva, em virtude de apresentarem agravamento/falência respiratória, ou outras problemáticas incompatíveis com uma ventilação espontânea eficaz. Esta prática ventilatória está correntemente associada à administração de sedação que deverá ser gerida, com o intuito de minimizar a ansiedade, assim como promover o conforto, segurança e evitar a agitação dos doentes (Humphrey, Everhart, Kosmisky, \& Anderson, 2018).

Este é um processo complexo, motivo pelo qual se recomenda a utilização de escalas de sedação nas unidades de Cuidados Intensivos, em doentes com ventilação invasiva e sedados. Em Portugal, num estudo desenvolvido por Pinto (2011) constatou-se que são utilizadas escalas para a avaliação da sedação em 96\% das unidades, sendo a Escala de Ramsey a mais utilizada 
(80\%) e a menos utilizada (3\%), a Motor Activity Assessment Scale.

O nível de sedação de um doente ventilado pode ser classificado como ligeiro, moderado e profundo. A utilização de sedação profunda prolongada tem vindo a ser associada a diversas complicações, tais como delirium e tempos de ventilação mais prolongados, assim como sintomas associados a stress póstraumático e memórias mais perturbadoras da sua permanência no internamento (Tingsvik et al., 2013). Por outro lado, a sedação ligeira, pode acarretar vários riscos, nomeadamente extubação, queda, ou remoção de outros dispositivos terapêuticos adjuvantes ao tratamento (Urden, Stacy \& Lough, 2013).

As guidelines internacionais mais recentes têm vindo a encorajar a utilização de práticas de sedação que permitam que o doente com ventilação invasiva esteja acordado, consciente e interativo, sempre que possível e caso não haja contraindicações (Mehta, Spies, \& Shehabi, 2018). Nas últimas décadas, cuidar de doentes conscientes, submetidos a ventilação invasiva, tornouse habitual nas Unidades de Cuidados Intensivos escandinavas (Klarsson \& Bergbom, 2015). Nesta linha de pensamento, os cuidados prestados a este tipo de doentes constituem uma mudança e desafio na interação doente/enfermeiro (Mortensen, Kjaer, \& Egerod, 2019). Constatou-se também, no estudo destes autores, que o delirium é mais percecionado pelos enfermeiros quando os doentes ventilados estão conscientes.

A frustração pela disfuncionalidade na comunicação com estes doentes é salientada por estes profissionais que, no entanto, revelam apreço na interação com o alvo dos cuidados (Mortensen et al.,2019), comparativamente às situações em que o doente está em sedação profunda, não conseguindo comunicar, o que o impede de expressar emoções, dor ou desconforto. A interação dos enfermeiros com o doente ventilado invasivamente é indiscutivelmente indissociável da implementação de intervenções no âmbito comunicacional, mediante a utilização de várias estratégias comunicacionais (Ten Hoorn et al, 2016).

Ao cuidar de doentes com sedação ligeira, os enfermeiros desempenham um papel crucial na promoção da integridade, autonomia e privacidade dos mesmos, assim como na tentativa de assegurar um ambiente adequado à prática dos cuidados (Tingsvik et al., 2013). Estes cuidados requerem mais tempo, recursos, enfermeiros mais presentes (Mortensen et al., 2019) e maior dedicação (Backlund, Persson, \& Haziabdic, 2018), nomeadamente pelas dificuldades comunicacionais e risco mais elevado de remoção de dispositivos indispensáveis para o tratamento.

\section{METODOLOGIA}

Este estudo é qualitativo, de caráter exploratório. O método de recolha de dados que consideramos mais adequado para dar resposta ao objetivo pretendido foi o focus group. Esta escolha prendeu-se com o facto de, através da seleção de um grupo de indivíduos com características e temáticas em comum, se possibilitar a interação entre os mesmos, a partilha de ideias e o pensamento crítico, potenciando maior aquisição de dados de investigação. Selecionou-se uma amostra não probabilística e por conveniência, constituída por seis enfermeiros, com o mínimo de cinco anos de exercício profissional em Cuidados Intensivos, o que segundo Benner (2001), os coloca no nível de peritos. O critério de inclusão foi participarem no focus group apenas Enfermeiros Especialistas em Enfermagem MédicoCirúrgica, na área de enfermagem à pessoa em situação 
Doente sedado, consciente e ventilado invasivamente: terapêuticas de enfermagem

crítica, os quais, para além de peritos detêm vários domínios de competências avançadas que, aliadas à evidência científica e reflexão permitem tomadas de decisão, perante situações complexas de risco eminente de vida.

A colheita de dados decorreu em novembro de 2020. Foi efetuada uma gravação audio e vídeo do focus group, com a duração de 90 minutos, via a plataforma informática Microsoft Teams, em virtude das contingências associadas à pandemia por Covid-19. Posteriormente, realizou-se a transcrição verbatim do focus group, através do processador de texto Microsoft Word.

A análise dos dados foi realizada utilizando a metodologia de análise de conteúdo segundo Bardin (2015), sem categorização a priori, através da qual se procedeu à identificação das respetivas categorias e subcategorias.

Este estudo de investigação teve parecer favorável (Parecer no 015/2020). Foi solicitado aos participantes, o consentimento para integrar este estudo, assim como para gravação de áudio e vídeo da reunião. A garantia da confidencialidade foi assegurada pelo anonimato da identidade e dos dados obtidos, através da substituição do nome dos mesmos, por uma codificação ordinal.

\section{RESULTADOS/DISCUSSÃO}

Da amostra constituída por seis enfermeiros, constatase que, a maioria é do sexo feminino (67\%). Relativamente à categoria profissional, todos os participantes são especialistas em Enfermagem Médico-Cirúrgica. O tempo de exercício profissional varia entre os 12 e os 26 anos, sendo a média de 19,6 $( \pm 2,8)$ anos. Em relação ao tempo de exercício profissional em Cuidados Intensivos, varia entre os 11 e os 23 anos de experiência, com uma média de 16,8 ( \pm 3 ) anos.

Após análise do conteúdo da transcrição do focus group, foram identificadas cinco categorias de terapêuticas de enfermagem: Redimensionamento da vigilância, Gestão da terapêutica, Autocuidado, Implementação de estratégias comunicacionais e Implementação de estratégias face à agitação/desorientação.

\section{Redimensionamento da vigilância}

O redimensionamento da vigilância é um aspeto de extrema importância na abordagem ao doente ventilado mecanicamente, acordado e sob sedação ligeira. Este novo paradigma de sedação, advogado por um número crescente de profissionais e implementado em múltiplas Unidades de Cuidados Intensivos requer dos enfermeiros, um ajuste no processo cuidativo e uma atenção privilegiada face ao doente.

“... nós temos que ter uma vigilância muito mais apertada dos doentes..." (P6, novembro, 2020).

"...temos que fazer um acompanhamento muito mais próximo destes doentes" (P4, novembro, 2020).

“... antecipar alguns daqueles eventos que podem acontecer, porque são múltiplos os dispositivos que ele tem, não é? Esses dispositivos podem facilmente sair" (P2, novembro, 2020).

“Portanto (...) implica de nós uma observação mais (...) mais próxima e mais atenta" (P2, novembro, 2020).

No discurso dos participantes evidencia-se que, no cuidado a estes doentes é essencial uma maior presença dos enfermeiros, o que é corroborado por Karlsson, V., \& Bergbom, I. (2014) e Tingsvik et al (2013). É igualmente expresso pelos intervenientes, a premência de maior proximidade nas intervenções de enfermagem, assim como uma maior observação e vigilância, com o intuito de prevenir /antecipar 
Doente sedado, consciente e ventilado invasivamente: terapêuticas de enfermagem

possíveis eventos adversos, como a retirada dos dispositivos terapêuticos.

\section{Gestão da Terapêutica}

A gestão da terapêutica ajustada a cada doente é uma intervenção de enfermagem interdependente, indissociável de outras de caráter independente, constituindo um foco de atenção dos enfermeiros, em prol do bem-estar e conforto do doente.

A administração de analgesia requer uma avaliação adequada do doente, por parte do enfermeiro, assim como, a determinação do nível de sedação deve ser adequada a cada indivíduo (Urden et al., 2013).

"... é por aí que nós devemos caminhar e cada vez mais usar os protocolos de sedação" (P5, novembro, 2020).

“... quer a sedação, quer a analgesia também são prescritos por objetivos e ...e é verificado...se a analgesia está correta, se é suficiente para aquele doente, se ele está confortável, se não está confortável, através da aplicação das escalas; da escala da dor, da escala da RASS, da BPS" (P4, novembro,

2020).

“... temos que gerir muito bem, a...analgesia/sedação" (P1, novembro, 2020)

"Nós fazemos a gestão da analgesia e da sedação de, de uma forma autónoma, com base em fluxogramas que temos... Temos essa parte de autonomia na gestão da analgesia e sedação e gerimos para os objetivos" (P6 novembro, 2020).

“...temos muito a modalidade da analgesia multimodal...fazemos sempre dupla analgesia, no sentido de promover sempre...conforto ao doente, nomeadamente naquilo que se refere à dor..." (P2, novembro, 2020).

"Nós queremos sempre o doente o mais acordado e o mais colaborante possível, otimizando muito, muito a analgesia e fazemos sempre a analgesia preventiva podemos administrar de uma forma autónoma" (P6, novembro, 2020).

“... vamos vendo sempre a resposta em termos fisiológicos e em termos de verbalização do doente, para ver se ele pelo menos está confortável com todos os dispositivos aa, com esse tipo de sedação..." (P2, novembro, 2020). É verbalizado pelos participantes a incidência de intervenções, no âmbito da gestão da analgesia e da sedação, e a utilização de protocolos/fluxogramas específicos, ajustados a cada doente. Esta ideia é um aspeto igualmente enfatizado por diversos autores (Barr et al., 2013; Pinto, 2011; Teixeira, \& Durão, 2016). A administração e gestão de analgesia multimodal e de analgesia preventiva são também mencionadas nas unidades de registo deste focus group, como forma de evitar picos de intensificação de dor. Indo de encontro aos participantes, Mehta, Spies, \& Shehabi (2018) defendem que a utilização da analgesia multimodal de uma forma antecipada pode aumentar a sua eficácia. A necessidade premente de avaliação e vigilância criteriosa da dor é igualmente um aspeto enfatizado pelos enfermeiros, que mencionam a atenção dispensada à identificação e vigilância de sinais fisiológicos do 5o sinal vital, assim como, de sinais de desconforto do doente. Segundo Teixeira \& Durão (2016), a gestão da dor engloba a avaliação, monitorização e tratamento da mesma, mediante a utilização de escalas e indicadores de monitorização, passíveis de identificar a sua intensidade e permitir avaliar a eficácia das intervenções instituídas. As mesmas autoras, no estudo realizado, vão de encontro ao expresso pelos participantes, identificando indicadores de dor como: a inquietude, movimentos corporais, variação do diâmetro pupilar, assincronia do ventilador, vocalizações e expressão facial.

\section{Autocuidado}

O autocuidado dos doentes é foco dos cuidados de enfermagem à pessoa em situação crítica, pelo facto de esta, frequentemente, não ser capaz de tratar do que é necessário para se manter operacional. 
Doente sedado, consciente e ventilado invasivamente: terapêuticas de enfermagem

Num estudo desenvolvido por Klarsson \& Bergbom (2015) foi considerado pelos profissionais ser mais satisfatório cuidar de doentes ventilados, sedados e conscientes, em virtude de os poderem motivar e envolver nos cuidados.

$\mathrm{Na}$ categoria Autocuidado foram identificadas cinco subcategorias: Autocuidado mobilizar-se, Autocuidado posicionar-se, Autocuidado transferir-se, Autocuidado higiene, Promoção dos autocuidados.

\section{Autocuidado mobilizar-se}

A dependência do doente sedado e ventilado internado em cuidados intensivos no autocuidado mobilizar-se pode conduzir a diversas complicações. Neste sentido, de acordo com os enfermeiros que participaram no estudo, a possibilidade de haver alguma participação do mesmo na sua mobilidade constitui-se como um ganho em saúde.

"Em termos de mobilidade naquele doente o que vamos conseguir é diminuir o seu tempo de internamento, com uma recuperação mais rápida, logo há ganhos em saúde" (P4, novembro, 2020).

“... o facto de ele poder mexer as pernas poder mexer os braços, o pouco movimento que ele possa fazer com os pés, já vamos ganhar aí bastante..." (P4, novembro, 2020). “... que o doente consiga fazer o seu, a sua movimentação, a sua mobilização..." (P4, novembro, 2020).

\section{Autocuidado posicionar-se}

Segundo alguns participantes, o facto de o doente se encontrar consciente, torna muitas vezes possível, que o mesmo colabore nos seus posicionamentos.

“... até que nos ajude no posicionamento" (P4, novembro, 2020).

“... promover os posicionamentos...” (P1, novembro, 2020).

\section{Autocuidado transferir-se}

A prática de sedação ligeira nos doentes ventilados permite, de acordo com os participantes que o autocuidado transferir-se se realize com alguma participação do doente, facto que não seria possível se este se encontrasse num grau de sedação mais profunda.

“...incentivar o doente...os doentes que possam estar otimizados e que possam fazer levante...mesmo entubados e ventilados" (P4, novembro, 2020).

\section{Autocuidado higiene}

É relatado por um dos participantes do focus group que, na prestação de cuidados de higiene aos doentes conscientes, sedados e ventilados, os mesmos são gradualmente envolvidos neste autocuidado.

“... ponho uma esponja em cima do tórax, ponho a mão do doente em cima do tórax e uma em cima da esponja, $e$ basicamente a função dele é esfregar o tórax, passados uns dias...está também a prestar cuidados de higiene à cara, aos braços". (P2, novembro, 2020).

\section{Promoção dos autocuidados}

De acordo com o que aludem os enunciados descritivos dos cuidados especializados em Enfermagem MédicoCirúrgica (2017), o enfermeiro especialista nesta área deve implementar planos de cuidados individualizados, que, para além de outras vertentes contemplem a promoção do autocuidado dos doentes. Em consonância com este documento, a narrativa dos participantes revela que, cada vez mais as terapêuticas de enfermagem ao doente consciente, sedado e ventilado invasivamente têm grande incidência na promoção dos diversos autocuidados:

“... o autocuidado inicia-se muito precocemente e inicia-se muitas vezes no Serviço de Cuidados Intensivos" (P1, novembro, 2020). “... ao eles estarem acordados estão mais autónomos dentro das suas limitações e quer dizer que nós como enfermeiros, cada vez mais estamos a promover a autonomia... a nível de todos os autocuidados" (P5, novembro, 2020).

“...tentamos sempre que possível otimizar a que o doente colabore muito nos cuidados..." (P6, novembro, 2020). 
Doente sedado, consciente e ventilado invasivamente: terapêuticas de enfermagem estão a facilitar a comunicação do doente com o tubo" (P1,

\section{Implementação de estratégias comunicacionais}

A comunicação é um elemento-chave na interação enfermeiro-doente, que adquire particular relevância quando, por qualquer motivo, o doente encontra limitações a este nível.

Devido à incapacidade de comunicação verbal por parte do doente consciente, sedado e ventilado invasivamente, é imperioso ter experiência e criatividade para desenvolver estratégias comunicacionais, conducentes à satisfação das necessidades do mesmo.

“... uns quadros, arranjamos uns, uns livros com vários, com vários desenhos pra mostrar ao doente, por forma a melhorar a própria comunicação" (P6, novembro, 2020). "Nós há alguns anos atrás, já tínhamos implementado no nosso serviço quadro de vidatak para comunicação com o doente. Atualmente nós estamos a trabalhar, não sei se vocês conhecem o aplicativo icu patient comunicater?" (P1, novembro, 2020).

“... tínhamos o desafio de tentar a, arranjar a...estratégias, inclusive, para além da psicóloga, englobou uma terapeuta da fala, para alguns doentes em que nós não tínhamos mesmo forma de os entender" (P6, novembro, 2020).

"... nós temos quadros, quadros com imagens para eles agruparem ideias e temos também quadros com abecedário, para que eles possam constituir frases ou palavras, de forma a podermos comunicar" (P2, novembro, 2020). “... temos vindo a tentar desenvolver... a tentativa de leitura de lábios(sorriso), mesmo com o tubo inserido na boca, não é?" (P2, novembro, 2020).

"Tentamos ajustar a forma de comunicar com depois consoante o tipo de doente: se é um doente mais calmo, se é um doente mais agitado" (P2, novembro, 2020).

“... esse aplicativo é muito fácil de...conseguir escrever...até aparece a imagem do corpo e ele pode tocar onde lhe dói mais e ter a intensidade da dor...É uma das estratégias que novembro, 2020).

“... uma das intervenções que nós temos ...é ser o ele de ligação na comunicação”, "...nós pusemos o computador à frente, para ele falar com a filha e servimos nós de interlocutores entre a família..."(P1, novembro, 2020). Relativamente às estratégias referidas pelo grupo de participantes para comunicar com o doente, são múltiplas, nomeadamente a utilização de livros com desenhos, quadros com imagens e letras, leitura de lábios e aplicativos eletrónicos. Estes aplicativos e os quadros comunicacionais são igualmente referenciados na revisão da literatura realizada por Ten Hoorn, Elbers, Girbes, \& Tuinman (2016). Por outro lado, a formação realizada por alguns enfermeiros de cuidados Intensivos, no âmbito da psicologia e terapia da fala são enfatizados como importantes na dinamização da comunicação com o doente.

Um aspeto ainda referido foi o facto de estas estratégias requererem adaptação a cada doente, e de as mesmas constituírem um veículo para a expressão da sensação e intensidade da dor, por parte do mesmo.

A comunicação com a família é indissociável do processo comunicacional com o doente ventilado invasivamente. Como tal, os participantes expressaram o seu papel enquanto interlocutores com a família, através da utilização do computador, como uma das estratégias.

\section{Implementação de estratégias face à agitação/desorientação}

A agitação e desorientação são conceitos frequentemente interligados e presentes nos doentes internados em Cuidados Intensivos, particularmente naqueles privados da comunicação verbal e cujo grau de sedação lhes permite observar o complexo meio onde estão inseridos. Estes sinais podem ter várias 
Doente sedado, consciente e ventilado invasivamente: terapêuticas de enfermagem

causas associadas, sendo o papel do enfermeiro fundamental para minimizar a sua ocorrência.

Face à agitação e desorientação destes doentes foram identificadas pelos enfermeiros, várias terapêuticas de enfermagem, não farmacológicas. As mesmas foram englobadas em três subcategorias: Gestão da agitação, Promoção do sono e repouso e Regulação do ritmo circadiano.

Na Gestão da agitação foram referidas terapêuticas como a maior presença do enfermeiro, a massagem e, no caso de mais nenhuma estratégia ser capaz controlar o quadro de agitação é mencionada a utilização da restrição física.

“... acho que essa presença, o facto de, de estar presente às vezes acalma o doente. Às vezes não é só a terapêutica...às vezes o estar ali e perceber o que é que o doente quer..."(P1,

novembro, 2020).

"... aquela massagem é muito importante para minimizar esses quadros agitativos..." (P1, novembro, 2020).

"Naquele doente que de todo, quando não funciona nada, acabamos sim, por utilizar restrição física, em último caso, sempre" (P2, novembro, 2020).

Ao incidir na Promoção do sono e repouso, assim como na Regulação do ritmo circadiano, foram apontadas pelos intervenientes do estudo, estratégias que se enquadram conjuntamente nestas subcategorias, tais como:

\section{-Gestão da luminosidade da unidade}

"As luzes, tentamos sempre que possível minimizar a partir da meia noite, uma da manhã...tentar minimizar a intensidade da luz"(P2, novembro, 2020).

“... em vez de acender as luzes de toda a unidade...só acendemos metade...e isso minimizou alguns quadros de agitação que tínhamos durante a noite..." (P1, novembro, 2020).

\section{- Utilização de vendas oculares durante a noite}

“...também a aquisição...daquelas olheiras que a gente tem às vezes nos aviões" (P2, novembro, 2020).

- Controle do ruído, através do ajuste dos alarmes dos ventiladores e monitores

" utilização de tampões auriculares e instalação de dispositivos avaliadores do ruído"(P2, novembro, 2020).

"..tentar minimizar os apitos dos ventiladores e dos monitores" (P2, novembro, 2020).

"Já adquirimos tampões para...auriculares para alguns doentes, no sentido de minimizar a... o ruído" (P2, novembro, 2020).

“... adquirimos...os tampões para colocar nos doentes, porque...muitos doentes acordam a meio da noite a pensar que é de manhã... acordam agitados, e não sabem onde estão, não sabem que horas são, pensaram...que a noite já, já terminou" (P1, novembro, 2020).

“... instalamos um dispositivo para avaliação do ruído",

"...tentar adaptar a monitorização dos alarmes de cada doente..." (P6, novembro, 2020).

Este conjunto de estratégias expostas vai de encontro ao resultado de estudos que abordam a utilização de máscaras para os olhos e tampões auriculares, na promoção do sono dos doentes, internados em Unidades de Cuidados Intensivos (Vieira, Ferreira, \& Goes, 2018). A utilização de tampões auriculares é também mencionada no estudo desenvolvido por Hofhuis et al. (2018). Estes autores corroboram o discurso dos participantes, identificando a redução do volume dos alarmes dos ventiladores, como outra estratégia facilitadora do sono dos doentes. A luminosidade e o ruído são fatores que também influenciam a regulação do ritmo circadiano do doente em ambiente de cuidados intensivos. Neste sentido, Tovar (2017), tal como os participantes conclui que, a adoção de estratégias conducentes à redução da 
intensidade da luz e do ruído são medidas adotadas pelos enfermeiros.

\section{CONCLUSÃO}

No processo de transição saúde-doença do doente sedado, consciente e ventilado invasivamente, o papel do enfermeiro de cuidados intensivos é essencial. Os achados deste estudo permitiram identificar diversas terapêuticas de enfermagem valorizadas pelos enfermeiros, no cuidado a estes doentes. Estas focamse na maior necessidade de incremento da vigilância dos doentes, associada a uma maior proximidade e presença; na gestão da analgesia/ sedação, mediante a vigilância/avaliação de sinais de dor e utilização de escalas; na promoção do potencial de autocuidado; na implementação de estratégias facilitadoras da comunicação com o binómio doente/família e de estratégias para gestão dos quadros de agitação/desorientação do doente. A elaboração deste estudo permitiu-nos constatar que este novo paradigma de sedação ligeira dos doentes possibilita que, cada vez mais o enfermeiro envolva o doente nos cuidados, tendo em vista a promoção precoce do seu potencial de autonomia, com o consequente impacto significativo na recuperação do mesmo e obtenção de ganhos em saúde. Como limitação deste estudo foi identificada a inexistência de estudos de investigação com a mesma temática. Para desenvolvimentos futuros consideramos ter interesse ampliar a investigação efetuada a uma amostra constituída por enfermeiros peritos de Unidades de Cuidados Intensivos de outras áreas do país, a fim de ser possível a comparação de resultados.

\section{REFERÊNCIAS BIBLIOGRÁFICAS}

Bäcklund, K., Persson, K., \& Haziabdic, E. (2018). Intensive Care Nurses' Experiences of Caring for Intubate Patients under Light Sedation: A Qualitative Study. Open Journal of Nursing, 8, 473 - 484.

Bardin, L. (2015). Análise de Conteúdo. Lisboa: Edições 70.

Barr, J., Fraser, G., Puntillo, K., Ely, E., Gélinas, C., Dasta, J., ..., \& Jaeschke, R. (2013). Clinical Practice Guidelines for the Management of Pain, Agitation, and Delirium in Adult Patients in the Intensive Care Unit. Critical Care Medicine, 41(1), 263-306. https://doi.org/10.1097/CCM.0b013e3182783b72

Benner, P. (2001). De Iniciado a Perito. Coimbra: Quarteto.

Hofhuis, J., Rose, L., Blackwood, B., Akerman, E., McGaughey, J., Egerod, I., ... \& Spronk, P. (2018). Clinical practices to promote sleep in the ICU: A multinational survey. International Journal of Nursing Studies, 81, 107 - 114. https://doi.org/10.1016/j.ijnurstu.2018.03.001

Humphrey, M., Everhart, S., Kosmisky, D., \& Anderson, W. E. (2018). An evaluation of patient-specific characteristics on attainment of target sedation in an intensive care unit. Heart \& Lung, 47(4), 387391. https://doi.org/10.1016/j.hrtlng.2018.05.008

Klarsson, V.,\& Bergbom, I. (2015). ICU Professionals' Experiences of Caring for Conscious Patients Receiving MVT. Western Journal of Nursing Research, 37(3), 360375. https://doi.org/10.1177/0193945914523143

Mehta, S., Spies, C., \& Shehabi, Y. (2018). Ten tips for ICU sedation. Intensive care medicine, 44(7), 11411143. https://doi.org/10.1007/s00134-017-4992-9

Meleis, A. (2010). Transitions Theory. Middle Range and Situation Specific Theories in Nursing Research and Practice. New York: Springer Publishing Company.

Meleis, A., Sawyer, L., Im, E., Hilfinger, D. ,\& Schumacher, K. (2000). Experiencing Transitions: An Emerging Middle_Range Theorys. Advances in Nursing Science, $23 \quad$ (1), 12-28. https://doi.org/10.1097/00012272-200009000-00006

Mortensen, C., Kjaer, M.,\& Egerod, I. (2019). Caring for non-sedated mechanically ventilated patients in ICU: $A$ qualitative study comparing perspectives of expert and competent nurses. Intensive and Critical Care Nursing, 52, 35-41. https://doi.org/10.1016/j.iccn.2019.01.004

Mota, L., Bastos, F., \& Brito, M. (2018). A pessoa submetida a transplante de fígado: terapêuticas de 
enfermagem no follow up. Revista de Enfermagem Referência, série IV, 16, 19-28

Ordem dos Enfermeiros (2017). Padrões De Qualidade dos Cuidados Especializados em Enfermagem MédicoCirúrgica: na área de Enfermagem à Pessoa em Situação Crítica, na área de Enfermagem à Pessoa em Situação Paliativa, na área de Enfermagem à Pessoa em Situação Perioperatória e na área de Enfermagem à Pessoa em Situação Crónica (Assembleia Extraordinária do Colégio da Especialidade de Enfermagem MédicoCirúrgica). Retirado de em: https://docplayer.com.br/88218153-Assembleiaextraordinaria-do-colegio-da-especialidade-deenfermagem-medico-cirurgica.html

Ordem dos Enfermeiros (2018). Competências Específicas do Enfermeiro Especialista em Enfermagem em Pessoa em Situação Crítica (Regulamento n.o 429/2018). Diário da República, 2aㅡ série, n.o 135 de 16 de julho de 2018, p. 19359-19360.

Pinto, F. (2011). Sedação e analgesia em unidades de cuidados intensivos em Portugal - resultados de um inquérito nacional (Tese de Mestrado). Instituto de Ciências Biomédicas Abel Salazar: Porto.

Teixeira, J., \& Durão, M. (2016). Monitorização da dor na pessoa em situação crítica: uma revisão integrativa da literatura. Revista de Enfermagem Referência, série IV, 10, 135-142. http://dx.doi.org/10.12707/RIV16026
Ten Hoorn, S., Elbers, P.W., Girbes, A.R., \& Tuinman, P.R. (2016). Communicating with conscious and mechanically ventilated critically ill patients: a systematic review. Critical Care 20, 333. https://doi.org/10.1186/s13054-016-1483-2

Tingsvik, C., Bexell, E., Andersson, A., \& Henricson, M. (2013). Meeting the challenge: ICU-nurses' experiences of lightly sedated patients. Australian Critical Care, vol. 26

124-129.

https://doi.org/10.1016/j.aucc.2012.12.005

Tovar, L. (2017). El Sueño en las Unidades de Críticos. Comunicaciones Escritas presentadas al III Congreso Virtual de la Sociedad Española de Enfermería de Urgencias y Emergencias, Ciberrevista Enfermeria de Urgencias, 58, 1-3.

Urden, L., Stacy, K., \& Lough, M. (2013). Cuidados Intensivos de Enfermagem (6a Ed.). Rio de Janeiro: Elsevier Editora, Lda.

Vieira, J., Ferreira, R., \& Goes, M. (2018). Protetores de ouvido e olhos na promoção do sono em Cuidados Intensivos. Revista de Enfermagem UFPE, 12 (10). https://doi.org/10.5205/1981-8963v12i10a236958p2784-2793-2018 